\title{
Valoración enfermera del estado emocional del paciente en hemodiálisis mediante la herramienta para la detección del estado emocional de pacientes en diálisis (EE-D)
}

\author{
Noelia Olmedo Moreno, Francisco Luis Sánchez Izquierdo, Nuria Urbón Peláez, Ana Rico González, Milagro \\ de La Caridad Abad Lambert, Joaquín González Miñano \\ Clínica de Hemodiálisis Asyter Albacete. España
}

\section{Resumen}

Introducción: Las alteraciones psicológicas y emocionales influyen en el estado bio-psico-social del paciente en hemodiálisis. Un mejor conocimiento de estos aspectos nos ayuda a la comprensión y al manejo de las situaciones difíciles en su día a día.

Objetivos: Con este estudio se describe el estado emocional de los pacientes en hemodiálisis por medio del cuestionario EE-D, aplicado por profesionales de enfermería y analizaremos si existe relación entre éste y otras variables individuales.

Metodología: Se realizó un estudio descriptivo de corte trasversal, a 84 pacientes en hemodiálisis. Se utilizó el cuestionario EE-D, donde se valora la tristeza y el nerviosismo percibido por el paciente en la última semana, sus preocupaciones en diferentes ámbitos y que motivaciones encuentra para sentirse mejor o ilusionarse. Se recopilaron en un cuestionario ad hoc, datos demográficos, laborales, de adhesión al tratamiento farmacológico, etc.

Resultados: En el parámetro tristeza obtuvimos una media de 3.8 (0 nada y 10 máxima), la moda fue 0 y un $73.8 \%$ obtuvo un nivel igual o inferior a 5 . En cuanto al nerviosismo, la media fue 3, la moda 0 y el $79.8 \%$ manifestaron un nivel igual o inferior a 5 .

En cuanto a las preocupaciones casi la mitad de los pacientes refirió tener preocupaciones relacionadas con el ámbito familiar y con su enfermedad o trata-

Correspondencia:

Noelia OImedo Moreno

C/ Carretera de Jaén, 19 (cv Carmelitas)

02006 Albacete. España

E-mail: soyuncaptus@gmail.com miento, seguido por el ámbito emocional, laboral y religioso respectivamente.

Conclusiones: Nuestros pacientes presentan niveles bajos de tristeza, medios en cuanto a sus preocupaciones relacionadas con su enfermedad, mostrando en su mayoría facilidad para ilusionarse al encontrar motivaciones para ello.

\section{PALABRAS CLAVE}

- ALTERACIONES PSICOLÓGICAS

- ESTADO EMOCIONAL

- HEMODIÁLISIS

- HERRAMIENTA ENFERMERÍA

Nurse evaluation of the patient's emotional state on hemodialysis using the tool for the detection of the emotional state of patients on dialysis (EE-D)

\section{Abstract}

Introduction: Psychological and emotional alterations influence the bio-psycho-social state of the patient on hemodialysis. A better understanding of these aspects helps to understand and manage difficult daily situations.

Objectives: This study describes the emotional state of patients on hemodialysis using the EE-D questionnaire, applied by nursing professionals and we will analyze if there is a relationship between this and other individual variables.

Methodology: A cross-sectional descriptive study was carried out in 84 patients on hemodialysis. The EE-D questionnaire was used, which evaluates the sadness 
and nervousness perceived by the patient in the last week, their concerns in different areas and what motivations they find to feel better or to be excited. Demographic, labor, adherence to pharmacological data, etc. were collected in an ad hoc questionnaire.

Results: In the sadness parameter, we obtained a mean of 3.8 ( 0 nothing and 10 maximum), the mode was 0 and $73.8 \%$ obtained a level equal to or less than 5. Regarding the concerns, almost half of the patients reported having concerns related to the family environment and their illness or treatment, followed by the emotional, occupational and religious environment, respectively.

Conclusions: Our patients present low levels of sadness and middle ones in the concerns related to their illness, showing in the majority, facilities to be excited, when finding motivations for it.

\section{KEYWORDS}

- PSYCHOLOGICAL ALTERATIONS

- EMOTIONAL STATE

- HEMODIALYSIS

- NURSING TOOL

\section{Introducción}

Cualquier patología orgánica que afecte a un individuo, produce en éste, alteraciones físicas propias de la enfermedad causante, desencadenando a su vez alteraciones psíquicas.

El individuo está enfermo y por ello sufre, como es lógico, la enfermedad renal no es una excepción y en ella como en las demás enfermedades, este sufrimiento debe de ser entendido como una serie de sensaciones, emociones, sentimientos y reacciones psicosomáticas añadidas a la enfermedad orgánica real.

La psicología aplicada a la nefrología es la adecuación de los conocimientos psicológicos, para una mejor y más completa comprensión del enfermo crónico, en concreto aquel bajo tratamiento de hemodiálisis (HD) que presenta en su diagnóstico y tratamiento, factores emocionales que causan dificultades en su vida.
Las enfermedades crónicas de tipo renal, no solo alteran al paciente por el proceso de adaptación que ello implica, si no que pueden producir además dificultades económicas, afectaciones en la autoestima y en las relaciones con los miembros de la familia y amigos $^{1}$.

Si a la sintomatología orgánica, a las limitaciones impuestas por la insuficiencia renal crónica y al tratamiento de hemodiálisis le añadimos un ritual casi diario y repetitivo durante meses e incluso años de su vida, dependiendo de una máquina, personal sanitario, molestias físicas, dificultades laborales y sociales, mala comprensión de la enfermedad por el entorno, limitaciones alimenticias, etc. desencadenarán en los pacientes alteraciones patológicas tales como depresión, ansiedad o estrés ${ }^{2}$.

Para hacer un abordaje psicológico necesitamos tener en cuenta diferentes aspectos tales como la educación, familia, sexo, edad, profesión, vivienda, alimentación y tratamiento, aportando una mejor y más completa visión del enfermo renal crónico³.

Cualquier enfermedad crónica que pueda ocasionar la muerte, crea un estado de rabia, además de añadir un factor de estrés al saber que en el caso de pacientes en HD su vida está ligada a una máquina de por vida, dando lugar a cambios de carácter negativos e inesperados, permanentes y estables.

El tipo de emoción y su intensidad, es el resultado de la interpretación que cada persona hace de la situación y de los recursos con los que cuenta para enfrentarse a la misma, o de las posibilidades que cree de tener éxito o fracaso, esto siempre va a variar de un paciente a otro dependiendo de la valoración cognitiva que realice sobre el impacto de la enfermedad renal y la HD, sobre sus condiciones de vida.

Convertirse en un enfermo crónico supone un impacto que afecta a la autoimagen y a la sensación de inseguridad por falta de control sobre los acontecimientos, además de requerir un periodo de afrontamiento donde las respuestas emocionales del enfermo están relacionadas con la ansiedad y las alteraciones del ánimo ${ }^{4}$.

Muchos de los síntomas físicos asociados a la ansiedad y depresión son confundidos con la sintomatología de la insuficiencia renal crónica, por esto, es importante realizar una evolución integral que no sólo involucre un exhaustivo examen médico, sino que también abarque un diagnóstico psicológico a través del cual poder abordar 
esta problemática, teniendo como objetivo ayudar a los pacientes a afrontar la enfermedad y su tratamiento ${ }^{1}$.

La ansiedad suele ser la respuesta emocional normal en las primeras etapas de adaptación a la HD. Además de estar relacionada con el tratamiento, también lo está con la incertidumbre respecto al futuro. La depresión también ha sido identificada como uno de los problemas clave del enfermo terminal. El miedo o preocupación es la emoción habitual más asociada.

En cuanto a las alteraciones conductuales, cabe destacar los comportamientos de autocuidado y hábitos adecuados, además de los relacionados con el estilo de vida, actividad y ejercicio físico.

La importancia del apoyo familiar y social está relacionado con el cuidado y la adhesión al tratamiento que muestra un paciente, si logramos que mantengan una vida activa ayudaremos a relativizar la enfermedad.

Queda demostrado, por diversos estudios, que el conocimiento de los aspectos psicológicos de los enfermos en HD, ayuda a la comprensión y al manejo de las situaciones difíciles con la que se enfrentan estos pacientes.

Por todo ello, el objetivo del estudio fue describir el estado emocional de los pacientes de HD por medio del cuestionario para la detección del estado emocional de pacientes en diálisis (EE-D) aplicado por profesionales de enfermería, analizando si existe relación entre el estado emocional de los pacientes con otras variables individuales.

\section{Material y Método}

Se realizó un estudio cuantitativo, descriptivo y de corte trasversal, a todos los pacientes en programa de HD de dos centros periféricos de Albacete y Cuenca. Se excluyeron pacientes con problemas de comunicación y alteraciones cognitivas. La muestra final fue de 84 pacientes.

Para medir el estado emocional de los sujetos, se utilizó el cuestionario EE-D, elaborado por García H, Rodríguez R. y grupo SEDEN. Es un formato de entrevista semiestructurada que consta de 5 ítems. En el primero se mide el nivel de tristeza y nerviosismo percibido por el paciente en la última semana en una escala del $0 \mathrm{a}$ 10 (donde 0 es nada y 10 es máximo). Otro dicotómi- co sobre las preocupaciones del paciente en diversos aspectos, tales como el familiar, laboral, emocional y religioso, sobre el tratamiento o enfermedad y la relación con los profesionales sanitarios. Dos ítems abiertos, donde se valoran si encuentran motivaciones para sentirse mejor o ponerse contento y un último campo, donde el profesional debe indicar si aparecen algunos síntomas o situaciones relacionados con desadaptación y malestar. Además en un cuestionario ad hoc se recopilaron datos demográficos, de adhesión al tratamiento farmacológico y si el paciente cree estar en lista de trasplante.

Las entrevistas se llevaron a cabo durante cuatro semanas por un grupo de enfermeras de cada centro durante el mes de marzo del 2016. Previamente se realizaron dos reuniones donde se unificaron criterios para la recogida de datos y se realizaron simulacros guiados por una psicóloga, sobre la aplicación de esta herramienta.

Todos los participantes fueron informados de la naturaleza y los objetivos del estudio, solicitándoseles, en ese momento, el consentimiento escrito y garantizándose siempre la confidencialidad de los datos y anonimato de los pacientes encuestados.

Los resultados de las variables cualitativas se expresan mediante frecuencias, porcentajes y moda, mientras que las cuantitativas se muestran como media \pm desviación estándar. Para estudiar la relación entre las variables cualitativas se utiliza la chi-cuadrado de Pearson. Se consideró estadísticamente significativo un valor de $p<$ de 0,05.

\section{Resultados}

Entre nuestros resultados en el cuestionario ad hoc, encontramos una distribución de los pacientes según su estado civil: un $64.3 \%$ están casados, $19 \%$ solteros, $10.7 \%$ viudos y un $6 \%$ divorciados. Según con quien conviven, obtenemos que un $15.5 \%$ de los pacientes viven solos. Sobre el nivel de estudios, un $21.4 \%$ manifiestan tener estudios de grado medio y un $8.4 \%$ estudios superiores. Del total de la muestra, un $6 \%$ refiere estar trabajando. Cuando preguntamos a los pacientes cual era su nivel de cumplimiento dietético, la media obtenida es de 6.5 sobre 10 y en cuanto al nivel económico se obtuvo un 5.5 de media. Respecto al tratamiento farmacológico un $85.7 \%$ dijeron conocerlo correctamente. Un $35.7 \%$ creia estar en la lista de trasplante y en el ámbito espiritual un $72.6 \%$ afirmaba tener creencias religiosas. 
Valoración enfermera del estado emocional del paciente en hemodiálisis mediante la herramienta para la detección del estado emocional...

El esfuerzo medio que manifestaron los pacientes para acudir a sesión de HD fue de 4.1; Ilama la atención que la moda fuera 0 con un $26.2 \%$ de las respuestas. Finalmente, el nivel de dependencia percibido por los pacientes fue 4 , siendo 0 la moda con un $28.6 \%$.

Con respecto al cuestionario $E E-D$, cuando preguntamos a los pacientes cual había sido su nivel de tristeza en la última semana, obtuvimos una media de 3.8, $(27.4 \%)$ y un $73.8 \%$ tuvo un nivel de tristeza igual o inferior a 5. En cuanto a los niveles de nerviosismo, la media fue 3, (38.1\%) y un $79.8 \%$ manifestó un nivel de nerviosismo igual o inferior a 5 .

En cuanto a las preocupaciones, un $48.8 \%$ refirió algún problema en el ámbito familiar, un $47,6 \%$ sobre su enfermedad o tratamiento, un $38.1 \%$ en el emocional, siendo las menos frecuentes en el ámbito laboral con un $16.7 \%$ y en el espiritual con un $4,8 \%$.

En cuanto a la pregunta sobre qué aspectos ayudan al paciente a sentirse mejor desde que está en tratamiento de HD, obtuvimos que un $73.8 \%$ de los pacientes coinciden en que existen motivaciones para ello, siendo los más repetidos la familia con un $20.2 \%$, el personal sanitario con un $9.5 \%$, ante la idea de un futuro trasplante renal con un $8.3 \%$ y los compañeros de tratamiento con un $5.9 \%$.

En cuanto a la facilidad para ilusionarse un $85.7 \%$ encontró alguna motivación por la que ilusionarse desde que están en $\mathrm{HD}$, siendo los aspectos más repetidos la familia un $26 \%$, el trasplante renal y el personal sanitario un $12 \%$ cada uno y los compañeros de tratamiento con un $11 \%$.

En cuanto a la parte observacional por parte del entrevistador, resultó que mientras se realizó la encuesta presentaban expresión facial de malestar un $19 \%$ de los pacientes. Además, se identificó un aislamiento desadaptativo en un $16.8 \%$ y un comportamiento demandante en un $17.1 \%$.

Al analizar la posible relación de las variables, encontramos asociación estadísticamente significativa entre:

- Pacientes que dicen tener preocupaciones emocionales / psicológicas con los pacientes que no encuentra motivaciones para sentirse mejor $(p=0.018)$.

- También encontramos relación entre los pacientes que no consideran la enfermedad / tratamiento como una preocupación con los pacientes que tienen menor capacidad para ilusionarse $(p=0.020)$.
Se observó relación, aunque no estadísticamente significativa, entre los pacientes con creencias religiosas y los que tienen más capacidad para ilusionarse $(p=0.058)$.

\section{Discusión}

Partimos de que en este estudio hemos usado una nueva herramienta para la enfermería de HD. Así nos ha resultado imposible encontrar antecedentes del uso de esta herramienta en nuestro ámbito.

Hemos asociado los niveles de tristeza y nerviosismo que nosotros preguntamos en el cuestionario EE-D con otros estudios donde se analizaron datos sobre depresión y ansiedad, obteniéndose similares resultados. Alvarez et al. plantean que los pacientes en tratamiento de HD presentan frecuentemente "trastornos emocionales adaptativos mixtos" conformados por la presencia conjunta de ansiedad y depresión ${ }^{5}$.

En estudios como el de "Evaluación de la depresión y ansiedad en pacientes con IRC sometidos a HD" realizado por Belkys J. et al, aparecen niveles de depresión valorados con Examen Mental y Escala de Hamilton para depresión que están en $26.2 \%{ }^{6}$, valores muy aproximados a nuestro estudio. En cambio, en "Insuficiencia renal y depresión" una revisión bibliográfica sobre el tema realizado por Orellana M. y Munguía A. se refiere que entre un $20 \%$ y un $100 \%$ de los pacientes de HD manifiestan depresión ${ }^{7}$, márgenes más altos que los nuestros. También, según Amador Coloma R. en el estudio "Depresión y ansiedad en HD: la creatividad para combatirlas" obtiene niveles más altos que los nuestros, donde el $54.24 \%$ de los pacientes de su muestra tienen algún grado de depresión ${ }^{8}$.

Por otro lado, en cuanto a los niveles de ansiedad en otros estudios, de nuevo se ven reflejados nuestros resultados en pacientes de HD. En "Ansiedad y depresión en pacientes con insuficiencia renal crónica en tratamiento de diálisis" por Amelia E. Paéz et al, un 23.3\% presentan altos niveles de ansiedad9, que podría extrapolarse a los niveles medio-alto de nerviosismo que mostraban el $20.2 \%$ de nuestros pacientes.

Para finalizar, hay que tener en cuenta que la principal limitación de este estudio fue la escasa bibliografía sobre la herramienta a estudio, así como el sesgo que implica que el personal de enfermería que trabaja habitualmente en el centro fuese el mismo que realizó la encuesta. 
Podría ser interesante que en futuras investigaciones se estudie la correlación de este cuestionario con otros ampliamente validados, con el fin de contrastar su fiabilidad para detectar posibles problemas o alteraciones y poder actuar sobre ellos.

En nuestra experiencia con el cuestionario EE-D, destacamos que nos pareció práctico, accesible y útil para detectar posibles alteraciones emocionales o de otro tipo por medio de profesionales de enfermería en nuestros pacientes en HD. Además, nos permitió obtener una mejor valoración del paciente para así poder informar al equipo interdisciplinar y ayudar a mejorar la calidad de vida de nuestros pacientes. A partir de toda esta información recogida, podemos hacernos una idea de que recursos personales utiliza el paciente para hacer frente a la enfermedad.

En conclusión, y a la vista de nuestros resultados, podemos afirmar que nuestros pacientes presentan niveles bajos de tristeza y medios en cuanto a sus preocupaciones relacionadas con su enfermedad, mostrando en su mayoría facilidad para ilusionarse al encontrar motivaciones para ello.

Recibido: 11 noviembre 16

Revisado: 13 noviembre 16

Modificado: 10 enero 17

Aceptado: 15 enero 17

\section{Bibliografía}

1. M. Á. Calvo y J. Cuenca. Significado de la primera hemodiálisis para el paciente renal y factores que influyen en su vivencia. Enferm Nefrol 2011: 14, (1):173-7.

2. C. A. Fernández y A. Bastos Flores. Intervención psicológica en personas con cáncer. Clínica contemporánea 2011: 2 (2): 187-207.

3. T. Rudnicki. Sol de invierno: Aspectos emocionales del paciente renal crónico. Diversitas, 2006: 279288.

4. G. C. Pérez Factores psicosociales y calidad de vida en pacientes con insuficiencia renal crónica. Rev Inves Psico: 69.

5. F. Álvarez- Ude, P. Rebollo, A. Vázquez, M. Fernández- Reyes, C. Mon y R. Sánchez. Síntomas físicos y trastornos emocionales en pacientes en programa de hemodiálisis periódicas. Nefrol, 2001: 21, (2):191-199.

6. B. J. Atencio Atencio, E. Nuccete Rios, J. Colina Chourio, H. Sumalave, F. Gómez Atencio y D. Hinestroza Finol. Evaluación de la depresión y ansiedad en pacientes con insuficiencia renal crónica sometidos a hemodiálisis. Archivos venezolanos de psiquiatría y neurología. 2004: 50(103):35-41.

7. M. Orellana y A. Munguía. Insuficiencia renal y depresión. Revista del postgrado de psiquiatría.2008: 1 (3) 10-12.

8. Amador Coloma R., Pons Raventos E. y Espinosa Calderón C. Depresión y ansiedad en hemodiálisis: la creatividad para combatirlas. Comunicaciones Presentadas al XXXIV Congreso Nacional SEDEN; 2009. p. 30-35.

9. A. Páez, J. Marcos y M. Á. de Bortoli. Ansiedad y depresión en pacientes con insuficiencia renal crónica en tratamiento de diálisis. Universitas Psychologica, 8 (1):117-124 2009. 
ANEXO 1. Cuestionario ad hoc.

1. Técnica de diálisis actual:
a. HD
b. DP manual
c. DP automática

2. Programa de diálisis y turno:
d. Mañana
e. Medio día
f. Tarde

3. Fecha de inicio en diálisis.

4. Entrada en diálisis: urgencias-programado (ECA)-traslado de otra técnica.

5. Etiología ERC.

6. ¿inclusión en la lista de trasplante?

7. ¿Ha estado en otras técnicas de diálisis? Si. No. ¿Cuáles? ¿Cuánto tiempo?

8. ¿Cuánto esfuerzo le cuesta venir a diálisis? De 0 a 10 . Donde 0 es poco esfuerzo y 10 el máximo esfuerzo posible.

9. Estado civil.

10. ¿En qué grado crees que dependes de alguien? 0-10 (papel familiar/económico).

11. ¿Vive usted solo actualmente?

12. ¿Trabaja actualmente?

13. ¿Cuál es su nivel de estudios?

14. ¿Cuál diría que es su nivel socioeconómico? 0-10

15. ¿Cree conocer bien usted su medicación? 0-10

16. ¿Sigue la dieta recomendada habitualmente? 0-10

17. ¿Tiene algún tipo de creencia espiritual o religiosa? Si /No. ¿Cuál?

18. Tipo de acceso vascular. 


\section{ANEX0 2. Cuestionario EE-D.} Emocional de Pacientes en Diálisis (EE-D)

1. Marque con una " $x$ " el número que mejor describa cómo se ha sentido durante la semana pasada (entre 0 ="nada triste" y 10 ="extremadamente triste"; 0 ="nada nervioso" y $10=$ "extremadamente nervioso").

\begin{tabular}{|l|l|l|l|l|l|l|l|l|l|l|l|l|l|l|l|l|l|l|l|l|l|}
\hline $\mathbf{0}$ & $\mathbf{1}$ & $\mathbf{2}$ & $\mathbf{3}$ & $\mathbf{4}$ & $\mathbf{5}$ & $\mathbf{6}$ & $\mathbf{7}$ & $\mathbf{8}$ & $\mathbf{9}$ & $\mathbf{1 0}$ & $\mathbf{0}$ & $\mathbf{1}$ & $\mathbf{2}$ & $\mathbf{3}$ & $\mathbf{4}$ & $\mathbf{5}$ & $\mathbf{6}$ & $\mathbf{7}$ & $\mathbf{8}$ & $\mathbf{9}$ & $\mathbf{1 0}$ \\
\hline
\end{tabular}

2. (Completar por el profesional) Queremos valorar sus preocupaciones actuales para ver si podemos ofrecerle alguna ayuda.

\begin{tabular}{|l|c|c|c|}
\hline \multirow{2}{*}{\multicolumn{1}{|c|}{ ÁREA }} & \multicolumn{2}{|c|}{$\begin{array}{c}\text { iHAY ALGO QUE LE } \\
\text { PREOCUPE? }\end{array}$} & \multirow{2}{*}{ ¿QUÉ LE PREOCUPA? } \\
\cline { 2 - 3 } & Sí & No & \\
\hline Familiar & & & \\
\hline Laboral & & & \\
\hline Emocional y/o Psicológico & & & \\
\hline Espiritual y/o Religioso & & & \\
\hline La Enfermedad y/o Tratamientos & & & \\
\hline La relación con profesionales sanitarios & & & \\
\hline Otro & & & \\
\hline
\end{tabular}

3. Desde que está en diálisis ¿Qué cree que es lo que le ayuda o que le ayudaría a sentirse mejor?

4. Desde que está en diálisis ¿Qué cosas le hacen ilusión o le "ponen contento"?

5. (Completar por el profesional tratando de detectar síntomas o situaciones que puedan ser especialmente preocupantes) Señale si se observa alguno de los siguientes signos externos de malestar.

- Expresión facial que transmite malestar (Tristeza, miedo, hostilidad...) $\square$ sí $\square$ NO

- Aislamiento desadaptativo $\square$ sí $\square$ NO

(Mutismo, incomunicación, sensación de aburrimiento, duerme toda la sesión, no preguntas dudas)

- Demanda constante de compañía y atención de la enfermería $\square$ sí $\square$ NO

(Quejas reiteradas, pide glucemias, llama a la enfermera por síntomas no controlados intradiálisis, llama al teléfono de atención continuada en diálisis con mucha frecuencia...

- Alteraciones del comportamiento en la sala de diálisis / Unidad de diálisis peritoneal (DP) $\square$ sí $\square$ NO

(Llega impuntual a las sesiones/revisiones, quejas sobre el orden de entrada a la sala, gritos, amenazas, insultos, comportamiento hostil en la Unidad de DP...)

- Otros $\square$ sí $\square$ No ¿Cuál?

Observaciones:

Adaptado de: National Comprehensive Cancer Network. Distress Management clinical practice guidelines. J Natt Comp Cancer Network. 2003;1:344 -374. Maté J, Mateo D, Bayés, R, Bernaus M, Casas, C, González- Barboteo J, Limonero JT, López-Postigo M, Sigo A y Viel S. Elaboración y propuesta de un instrumento para la detección de malestar emocional en enfermos al final de la vida. 2009. Psicooncologia, (6), Num 2-3, 507- 518. Autores: Garcí-Llana H, Rodriguez-Rey R y el grupo SEDEN para el estudio del estado emocional de pacientes en diálisis (2015). 